\title{
Spuren organaler Mehrstimmigkeit in ungarischen einstimmigen Melodien. Erkenntnisse aus Gesangbüchern des 16.-18. Jahrhunderts
}

\author{
Ilona FERENCZI \\ Institut für Musikwissenschaft \\ Forschungszentrum für Humanwissenschaften \\ Ungarische Akademie der Wissenschaften \\ Táncsics Mihály u. 7, H-1014 Budapest, Ungarn \\ E-Mail: ferenczi.ilona@btk.mta.hu
}

(Angekommen: September 2016; angenommen: November 2016)

\begin{abstract}
Among the European countries, Hungary had the richest corpus of plainchant in vernacular in the $17^{\text {th }}$ century. The complete liturgy was put down with a musical notation for the first time in Gál Huszár's songbook (1574). The Eperjes Gradual (1635) acquired a special position among the Protestant graduals in Hungarian by being the only Lutheran gradual and the only one containing polyphonic pieces. A comparison of the Magne Deus-melody in both sources concludes that the differences of the two monophonic melodies can be interpreted as reminiscences of an organum-like polyphonic practice. This hypothesis is sustained by a series of examples including not only melodies of Gregorian origin but also Hungarian-texted monophonic hymns from the $16^{\text {th }}$ to the $18^{\text {th }}$ centuries.
\end{abstract}

Keywords: Monophony, traces of polyphony, organum, Gál Huszár's songbook (1574), Eperjes Gradual (1635), Cantus Catholici (1651, 1655), Tabulatura Vietoris (ca 1675), Kolozsvár hymnbook (1744)

In den deutschen lutherischen Gottesdiensten des 16. Jahrhunderts gab es ein vielfältiges vom Mittelalter geerbtes monophones volkssprachliches gregorianisches Repertoire. ${ }^{1}$ Im 17. Jahrhundert wird der deutschsprachige gregorianische Gesang

1. Darüber s. Adolf Boës, „Die reformatorischen Gottesdienste in der Wittenberger Pfarrkirche von 1523 an und die ,Ordenung der gesenge der Wittembergischen Kirchen“ von 1543/44“, Jahrbuch für Liturgik und Hymnologie 4 (1958/1959), 1-40; Der Altargesang, 1. Teil. Die einstimmigen Weisen. Handbuch der deutschen evangelischen Kirchenmusik. Nach den Quellen herausgegeben von Konrad Ameln, Christhard Mahrenholz und Wilhelm Thomas unter Mitarbeit von Carl Gerhardt. I. Band (Göttingen: Verlag von Vandenhoeck \& Ruprecht, 1941); „Deutsches Kirchenamt“ und „Deutsch-evangelische Messe“ in Thomas Müntzer Schriften und Briefe. Kritische Gesamtausgabe. Unter Mitarbeit von Paul Kirn herausgegeben von Günther Franz (Gütersloher Verlagshaus Gerd Mohn [1968]), 25-155, 157-343. 
zum größten Teil durch die mehrstimmigen Cantionalen ersetzt. ${ }^{2} \mathrm{Zu}$ Beginn des 17. Jahrhunderts erschien in Prag ein prachtvolles tschechisches lutherisches Gesangbuch mit einem bedeutenden Haupt- und Nebengottesdienst-Material gregorianischer Herkunft. ${ }^{3}$ Nach der Schlacht am Weißen Berg wurde jedoch keine tschechische Sammlung mehr veröffentlicht. Bei den sich der Reformation schnell anschließenden nördischen Völkern war die volkssprachliche Gregorianik nicht tief verwurzelt. ${ }^{4}$

Unter den europäischen Ländern weist Ungarn den reichsten Bestand an volkssprachlichen Gregorianik im 17. Jahrhundert auf. Anfangs waren nur einzelne Sätze bzw. liturgische Gattungen größerer Feste aus dem Lateinischen übersetzt worden. ${ }^{5}$ Erst im zweiten, großen Gesangbuch von Gál Huszár (1574) liegt die vollständige Liturgie der Haupt- und Nebengottesdienste mit volkssprachlicher Gregorianik vor. ${ }^{6}$ Unerwartet und zum Teil unerklärlich wird im 17. Jahrhundert das ganze Land mit volkssprachlichen gregorianischen Quellen geradezu überflutet. Gradual genannte, reichhaltige oder weniger inhaltsvolle, beinahe korrekte oder sehr fehlerhaft aufgezeichnete, verzierte oder wenig dekorative handschriftliche Gesangbücher, und ein zusammenfassender Gradual-Druck kennzeichnen die Jahrzehnte vor der sich allmählich verstärkenden Gegenreformation. ${ }^{7}$ Von den protestantischen Gradualen ungarischer Sprache ist das Eperieser Gradual aus 1635 nicht nur das einzige evangelisch-lutherische, ${ }^{8}$ sondern auch das einzige,

2. Vereinzelt kommen Stücke gregorianischen Ursprungs (vor allem Hymnen) auch in den Cantionalen vor; z. B. in dem unlängst in den Besitz der Ungarischen Evangelischen Kirche gelangten, vermutlich aus Sachsen stammenden Cantionale aus den 1620er Jahren.

3. Tobiáš Závorka Lipenský, Pjsnie Chwál Božských: To gest Spěwowé Swatij Cýrkewnij Stařij y Nowij z Gradualı̊, z Antyphonár̆o̊ y giných wssech Duchownjch Pjsnij Latinských y Cžeských..., 1606 und 1620 in Prag erschienen.

4. Vgl. Erkki Tuppurainen, „Finnische ,Affenmusik‘: Liturgische Handschriften aus dem 16. und 17. Jahrhundert“, Jahrbuch für Liturgik und Hymnologie 36 (1996/1997), 235-243.

5. Über die einzelnen gregorianischen Gattungen bzw. Feste in ungarischer Sprache aus dem 16. Jahrhundert siehe László Dobszay, ,A magyar Graduál-irodalom első emléke“ [Das erste Denkmal der GradualLiteratur in ungarischer Sprache], Magyar Könyvszemle 98 (1982), 100-112; Ilona Ferenczi, „Das Fragment aus Csíksomlyó als Reformationsdenkmal. Ungarische Präfationen und tropisierte Ite missa est-Sätze“, Studia Musicologica 44/1-2 (2003), 47-62; Ilona Ferenczi - Lea Haader, „Magyar nyelvű introitus-töredékek Marosvásárhelyröl (XVI. század közepe)“ [Introit-Fragmente in ungarischer Sprache aus der Mitte des 16. Jahrhunderts aus Neumarkt (Marosvásárhely, heute Târgu Mureş)], Zenetudományi Dolgozatok 2010, hrsg. von Gábor Kiss (Budapest: MTA Zenetudományi Intézet, 2011), 57-72. Für die liturgischen Morgengesänge von Márton Kálmáncsehi, die als Anhang zum ersten Gesangbuch von Gál Huszár (1560-1561) erschienen sind, siehe Fußnote 11.

6. Gál Huszár, A keresztyéni gyülekezetben való isteni dicséretek és imádságok, Komjáti 1574 [Lobgesänge und Gebete für die christliche Gemeinde, Komjáti 1574]. Neuausgabe mit einer Studie von Gabriella Hubert (Budapest: MTA Irodalomtudományi Intézet, 1986) (= Bibliotheca Hungarica Antiqua 13).

7. Über die Gregorianik in ungarischer Sprache siehe Ilona Ferenczi, ,Die ungarische Gregorianik im 16. und 17. Jahrhundert“, Jahrbuch für Liturgik und Hymnologie 32 (1989), 158-165; dies., „Ein Versuch zur Vereinheitlichung der Liturgie im Fürstentum Siebenbürgen in der ersten Hälfte des 17. Jahrhunderts“, in Sursum Corda. Variationen zu einem liturgischen Motiv. Für Philipp Harnoncourt zum 60. Geburtstag, hrsg. von Erich Renhart und Andreas Schnider (Graz: Akademische Druck- und Verlagsanstalt-Andreas Schnider Verlags-Atelier, 1991), 342-348.

8. Die meisten - mehr als zwanzig - Graduale sind ev.-reformiert, außerdem gehören zu dieser Buchgattung nur einige kleine unitarische Handschriften. 
welches mehrstimmige Stücke und sogar mehrstimmige Passionen enthält. Dadurch wird seine Sonderstellung noch mehr unterstrichen.

Als die Verfasserin gegenwärtigen Beitrages vor vielen Jahren die kritische Ausgabe des Eperieser Graduals vorbereitete, ${ }^{9}$ hielt sie die Korrekturen, die sie an den mit oder ohne Noten aufgezeichneten Sätzen vornahm, in den Anmerkungen genau fest. Da über die Hälfte der einstimmigen gregorianischen und Volksgesänge sowie der mehrstimmigen Sätze des Genfer Psalters, der deutschen Kantionen sowie die in Ungarn entstandenen Sätze fehlerhaft aufgezeichnet waren, wurde die Revisionsarbeit bald zur Routine. ${ }^{10}$ So schien auf den ersten Blick auch die Ausbesserung der Notation der populären Magne Deus-Melodie vonnöten zu sein, die im Eperieser Gradual den das Offizium schließenden Benedicamus-Text begleitet. Anders als im Gesangbuch von Gál Huszár (1574), wo sie sich in einer Gestalt vorfindet, die mit der mittelalterlichen Praxis übereinstimmt, ${ }^{11}$ biegt im Eperieser Gradual der Mittelschluss, statt des unteren $c$ zum oberen $c$ um, und zwar in der Weise, dass in der zweiten achtsilbigen Melodiezeile der Teil vom dritten bis zum siebenten Ton eine Quinte und der letzte Ton eine Oktave höher als die Hauptmelodie aufgezeichnet ist (Notenbeispiel 1). ${ }^{12}$

Die Korrektur ist jedoch unterblieben. Zur Vorsicht mahnte eine ähnlich sonderbare Variante der Melodie in dem 1655 herausgegebenen slowakischsprachigen Cantus Catholici Gesangbuch, in dem das betreffende Melodiesegment nicht in Benedicamus-Funktion, ${ }^{13}$ sondern als ebenfalls tropierter dritter Teil des Kyrie

9. Veröffentlicht als Graduale Ecclesiae Hungaricae Epperiensis 1635 [im Weiteren: Grad. Eccl. Hung. Epp.], hrsg. von Ilona Ferenczi (Budapest: MTA Zenetudományi Intézet, 1988) (= Musicalia Danubiana 9). Für eine überarbeitete Fassung des Vorwortes zu dieser Ausgabe siehe „Graduale Ecclesiae Hungaricae Epperiensis, 1635. Isoliert gebliebene Bemühung um die Erschaffung einer Kirchenmusik ungarischer Sprache“, Jahrbuch für Liturgik und Hymnologie 55 (2016), 216-228.

10. Die Mehrheit der Fehler resultiert aus der falschen Stellung des C-Schlüssels. Siehe das Kapitel „Musikalische Rechtschreibung“ in der Einleitung der Ausgabe sowie die Anmerkungen zu den einzelnen Sätzen: Grad. Eccl. Hung. Epp., 105-106 bzw. 187-202.

11. Huszár, A keresztyéni gyülekezetben való isteni dicséretek (1560-1561), f. XIVv, mit fehlerhafter Schlüsselsetzung und um eine Sekunde verrutschtem Mittelschluss. Zur mittelalterlichen Vorlage siehe Kyrie 78 in Ordinariums-Gesänge in Mitteleuropa. Repertoire-Übersicht und Melodienkatalog, hrsg. von Gábor Kiss (Kassel: Bärenreiter, 2009) (= Monumenta Monodica Medii Aevi Subsidia 6). - Unter den Morgengesängen von Márton Kálmáncsehi finden sich zwei Benedicamus-Texte ohne Noten, aber mit Verweis auf die Magne Deus-Melodie. Vgl. die Faksimile-Edition: Gál Huszár, A keresztyéni gyülekezetben való isteni dicséretek - Márton Kálmáncsehi, Reggeli éneklések (1560-1561), hrsg. von Béla Varjas, mit einer Studie von Gedeon Borsa (Budapest: Akadémiai Kiadó, 1983) (= Bibliotheca Hungarica Antiqua 12), f. a5b und f. d3a. Drei weitere Benedicamus kommen mit unterschiedlichen Melodien notiert vor; diese waren vermutlich weniger bekannte oder selten verwendete Melodien.

12. Grad. Eccl. Hung. Epp., Nr. 15; auf diese Melodie müssen auch die Texte Nr. 16, 17, 18, 19, 21, 22, 23, 25 und 26 gesungen werden. - In der gesamten Gradualliteratur sind nur diese beiden Quellen bekannt, in denen das Magne Deus-Melodie mit Benedicamus-Text vorkommt. Für die beiden Benedicamus-Textquellen siehe Répertoire de la poésie hongroise ancienne. Manuel de correction d'erreurs dans la base de données (im Weiteren: RPHA), hrsg. von Iván Horváth und Gabriella H. Hubert (Paris: Nouvel Objet, 1992), Nr. 1165 und 667.

13. Über die Benedicamus-Tropen in ungarischer Sprache siehe Ilona Ferenczi, „Tropus és kontrafaktum a 16-17. századi graduálokban“ [Tropus und Kontrafakt in den Gradualen des 16.-17. Jahrhunderts], Zenetudományi Dolgozatok 1999, hrsg. von Ágnes Gupcsó (Budapest: MTA Zenetudományi Intézet, 1999), $213-215$. 
Notenbeispiel 1a Die Magne Deus-Melodie im Gesangbuch von Gál Huszár (1574)

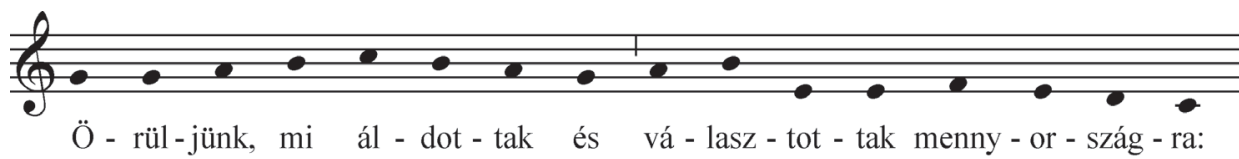

(Freuen wir uns, wir Gesegneten ...)



NotenbeisPiel 1b Die Magne Deus-Melodie im Eperieser Gradual (1635)

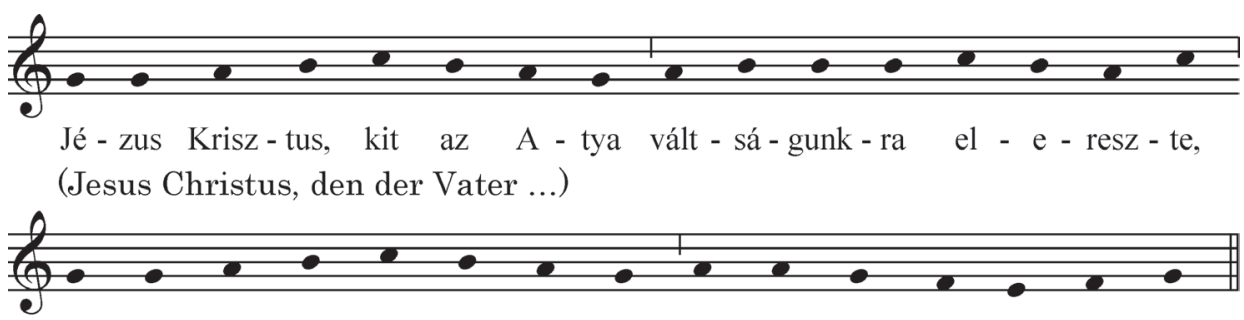

ké - rünk té - ged, légy szó - szó - lónk szent A - tyád - nak e - lőt - te.

de Ascensione Domini erscheint. ${ }^{14}$ Ähnlich gestaltet ist die Melodie im zweistimmigen Satz der Tabulatura Vietoris, die ebenfalls den Schlussabschnitt des dem Fest der Himmelfahrt zugeteilten Kyrie variiert. ${ }^{15}$ Wenn wir die Melodievariante des Eperieser Graduals, des slowakischen Cantus Catholici und der Tabulatura Vietoris über die Stammmelodie stellen, ergibt das Zusammenklingen des hervorgehobenen Abschnittes vor dem Mittelschluss ein mit Parallel- und dann mit Gegenbewegung ausgeglichenes Organum (Notenbeispiel 2).

Zwar hat sich bisher noch keine ungarische Aufzeichnung für die zweistimmige Organum-Variante der Benedicamus- oder Kyrie-Melodie des Magne Deus

14. Ferner erscheint es in der Kyrie-Melodie des allgemeinen Teils. Veröffentlicht als Cantus Catholici Nr. 115 und 148 in Ladislav Burlas - Ján Fišer - Antonín Hořejš, Hudba na Slovensku v XVII. storoči [Musik in der Slowakei im 17. Jh.] (Bratislava: Vydavatel'stvo Slovenskej Akadémie Vied, 1954). Die Beschreibung des slowakischen Cantus Catholici-Gesangbuches (1655) siehe Régi Magyarországi Nyomtatványok 3. 1636-1655 [Alte ungarische Druckwerke 3. 1636-1655], hrsg. von János Heltai et al. (Budapest: Akadémiai Kiadó, 2000), Nr. 2591, sowie Peter Ruščin, Cantus Catholici a tradícia duchovného spevu na Slovensku [Cantus Catholici und die Tradition des geistlichen Gesangs in der Slowakei] (Bratislava: Ústav hudobnej vedy SAV, 2012), 40-44.

15. Tabulatura Vietoris saeculi XVII, hrsg. von Ilona Ferenczi und Marta Hulková (Bratislava: Opus, 1986) (= Musicalia Danubiana 5), Nr. (209, 210) 211. - Diese „organale“ Variante der Magne Deus-Melodie blieb auch im 18. Jahrhundert erhalten. Im Kyrie-Satz der Deák-Szentes-Handschrift kommt diese Version sogar nicht nur im dritten, sondern auch im Eröffnungsteil vor, siehe A Deák-Szentes-kézirat. The Deák-Szentes Manuscript, hrsg. von Réka Kövári (Budapest: Magyarok Nagyasszonya Ferences Rendtartomány - MTA Bölcsészettudományi Kutatóközpont, 2013) (= Fontes Historici Ordinis Fratrum Minorum in Hungaria. Magyar Ferences Források 6), 199. 
Notenbeispiel 2 Melodievarianten im Gesangbuch von Gál Huszár (1574)

bzw. im Eperieser Gradual (1635), Cantus Catholici (1655) und Tabulatura Vietoris

(um 1675)

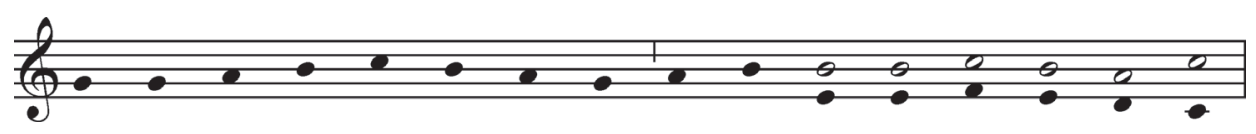

Notenbeispiel 3 Die Melodie in London, British Museum, Add. 27630

(mit schwarzen Noten)

bzw. in dem süddeutschen Kodex aus der zweiten Hälfte des 14. Jahrhunderts

(mit weißen Noten)

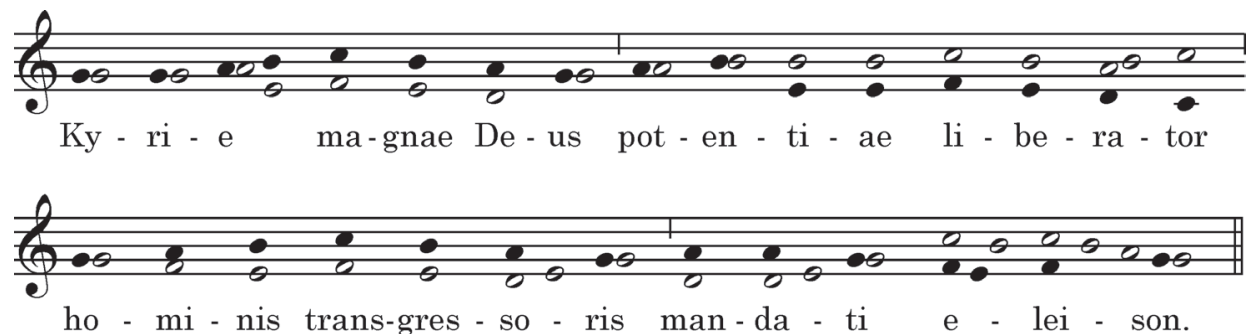

gefunden, ${ }^{16}$ doch ist zumindest vorauszusetzen, dass sich diese von der Hauptmelodie ausgehende und dann von ihr abweichende einstimmige Variante aus dem mehrstimmigen Klangerlebnis gespeist hat. Diese Hypothese unterstützen jene mittel- und westeuropäischen, vor allem in deutschen Handschriften erhaltenen zweistimmigen Organa, ${ }^{17}$ in deren mit Magne Deus-Text tropisierten Kyrie-Teilen über und unter der gregorianischen Melodie eine der obigen ähnelnde Ge-

16. Im zweistimmigen Kyrie-Satz der Tabulatura Vietoris erscheint nicht die Hauptmelodie als Begleitstimme unter der organalen Oberstimme. Gegenüber den Prim-, Quint- und Oktav-Zusammenklängen der zweistimmigen Organa sind in diesem Satz die Terz-Intervalle häufig. Weitere Beispiele mit der organalen Melodie von Magne Deus: Kyrie in Missa Alia Duplex 1. Class. und Missa XXXIV. De Corpore Christi in ORGANO MISSALE Fratris Patris Joannis Kaioni Ordinis Minorum Organistae et Organifabri Guardiani Conventus Mikhaziensis Anno 1667, hrsg. von Pál Richter (Csíkszereda: Státus Könyvkiadó, 2005) (= Csíksomlyói Ferences Kolostor Kincsei 3), 15, 434.

17. Die Aufzählung der Quellen siehe Die Handschrift London, British Museum, Add. 27630 (LoD), Teil I: Faksimile, Teil II: Übertragung der Organa und Motetten, hrsg. von Wolfgang Dömling (Kassel: Bärenreiter 1972) (= Das Erbe deutscher Musik 52, 53), Teil II, 71. Dömling veröffentlicht im Vorwort die Faksimiles des Kyrie magne Deus-Organum aus vier deutschen Quellen (Seite X, XI). Unter den hier angeführten Quellen kommen im RISM nur drei (zwei deutsche und eine österreichische) vor. Vgl. Handschriften mit mehrstimmiger Musik des 14., 15. und 16. Jahrhunderts, hrsg. von Kurt von Fischer und Max Lütolf (München-Duisburg: G. Henle Verlag, 1972) (= RISM B IV 3-4), 74, 435, 620. - Mit dem tropierten Organum-Satz des Kyrie magne Deus befassen sich Geering bzw. Göllner im Spiegel der erhaltenen Quellen; vgl. Arnold Geering, Die Organa und mehrstimmigen Conductus in den Handschriften des deutschen Sprachgebietes vom 13. bis 16. Jahrhundert (Bern: Paul Haupt, 1952) (= Publikationen der Schweizerischen Musikforschenden Gesellschaft 2), 1, 24, 42, 62; Theodor Göllner, Formen früher Mehrstimmigkeit in deutschen Handschriften des späten Mittelalters mit Veröffentlichung der Orgelspiellehre aus dem Cod. lat. 7755 der Bayer. Staatsbibliothek München (Tutzing: Hans Schneider, 1961) (= Münchner Veröffentlichungen zur Musikgeschichte 6), 26, 51-52. 
genstimme aufgezeichnet war; und sogar nicht nur in der ersten, sondern auch der zweiten Hälfte der Melodie. Die plagale mixolydische Hauptmelodie und die ähnliche Wendungen verwendende hinzugefügte Stimme des zweistimmigen Satzes kreuzen sich dreimal und verwenden damit die Technik des Stimmtausches (Notenbeispiel 3). ${ }^{18}$

Nachherige Untersuchungen stellten ähnliche Erscheinungen auch bei anderen einstimmigen Melodien aus dem 16.-18. Jahrhundert heraus, und zwar sowohl bei Sätzen gregorianischer Herkunft als auch bei Gemeindeliedern. Diese Funde möchten wir nun an einigen Beispielen darstellen.

Mit der Möglichkeit verborgener Mehrstimmigkeit in der Einstimmigkeit hat man bereits bei gregorianischen Melodien aus früheren Jahrhunderten gerechnet. Benjamin Rajeczky macht in seiner Beispielsammlung der mittelalterlichen mehrstimmigen Stile auf eine ähnliche Erscheinung aufmerksam. ${ }^{19}$ Als erstes Beispiel bringt er keine echte mehrstimmige Aufzeichnung, sondern zitiert verschiedenen Quellen entnommene Sequenzmelodien. Das letzte Strophenpaar der Sequenz von Pauli Bekehrung Sonent plausus laetabundi (Pelle nubem - Sis protector) schließt im Bakócz-Graduale und im Esztergomer Missale Notatum gleicherweise auf dem Grundton. Demgegenüber gestalten sich in demselben Verspaar im Futaki-Graduale die Melodiezeilen divergierend: Der b-Vers wiederholt im zweiten Abschnitt den a-Vers um eine Quinte höher. Damit weist er wahrscheinlich auf die Existenz einer zweistimmigen Form hin und erlaubt die Annahme, wonach „die sich in der Quinte wiederholenden Abschnitte der Sequenzen Gelegenheit bieten konnten, das Singen in parallelen Quinten einzuführen“. ${ }^{20}$

Der Organumstil erreichte an der Wende vom 12. zum 13. Jahrhundert seinen Gipfelpunkt mit dem Notre-Dame-Organum. Nach Rajeczky kann aufgrund der in Paris erworbenen musikalischen Erfahrungen der ungarischen Kleriker behauptet werden, dass die Organumpraxis schon im 12. Jahrhundert in Ungarn bekannt war. ${ }^{21}$ Diese Aufführungstechnik hat sich bis ins 14.-15. Jahrhundert im ganzen Land verbreitet, ihre Verwendung kann sogar bis ins 16. Jahrhundert ver-

18. Das zweistimmige Organum ist vereinfacht aus der Londoner Quelle wiedergegeben; erschienen: Die Handschrift London, f. 32, Nr. 29; der Satz gestaltet sich auch im nach dem Christe-Teil folgenden zweiten Kyrie (Kyrie homo natus) ähnlich.

19. Benjamin Rajeczky, ,Többszólamúság. A középkor polifón gyakorlata; Többszólamú források; Stílusok“ [Mehrstimmigkeit. Die mehrstimmige Praxis des Mittelalters; Mehrstimmige Quellen; Mehrstimmige Stilarten], in Magyarország zenetörténete I. Középkor [Musikgeschichte Ungarns I. Mittelalter], hrsg. von Benjamin Rajeczky (Budapest: Akadémiai Kiadó, 1988), 426-442; das diesbezügliche Beispiel auf Seite 433.

20. Ebd., 435. Rajeczky weist auf die Sequenzen-Ausgaben von A. Hughes und A. Moberg hin und fügt hinzu, dass in der ungarischen Praxis ,die in der Quintlage schließenden Sequenzen abschließend wahrscheinlich in der Quintparallele vorgetragen wurden“.

21. Ebd., 431-432. 
folgt werden. ${ }^{22}$ Auch später kann die Erscheinung nicht unbekannt gewesen sein, da sie sich nicht nur in der organalen Gesangspraxis, sondern auch in anderen musikalischen Stilen verbreitete. Die Parallel- oder Gegenbewegung der organalen Mehrstimmigkeit lässt sich in der schlichten Begleitung der Lieder aus dem 17. Jahrhundert ${ }^{23}$ oder in den Harmonieketten der Studentenmelodiarien des 18. Jahrhunderts erkennen. Deshalb ist die Behauptung vielleicht nicht allzu verstiegen, dass der einfache - mit der Zeit zunehmend primitiv wirkende - organale mehrstimmige Gesang sogar noch im 18. Jahrhundert in Ungarn eine lebendige Praxis war.

Aufgrund eines weiteren Beispiels in dem slowakischsprachigen Cantus Catholici (1655) lässt sich ebenfalls daran denken, dass zu der einstimmigen Melodie gregorianischer Herkunft (Hauptstimme, Vox Principalis) eine zweite Stimme (Vox Organalis) gesungen werden konnte. In den ersten Jahrzehnten des 17. Jahrhunderts wurden in den protestantischen Gradualen aufgezeichnet (und 1636 im Öreg Gradual gedruckt) die tropierten Sätze des Sanctus (Szent az Atya Isten, mindeneknek teremtöje - Heilig ist Gott Vater, der Schöpfer aller Dinge) und des Agnus Dei (Istennek báránya, Jézus Krisztus - Lamm Gottes, Jesus Christus), deren mittelalterliche Vorbilder in ungarischen, böhmischen und polnischen Quellen gleichermaßen erhalten sind. ${ }^{24}$ In den ungarischsprachigen Gradualen erscheint insgesamt achtmal jene Hauptmelodie aus unterschiedlich langen Zeilen, die nach dem Oktav-Mittelschluss nach unten biegend auf dem Grundton endet. ${ }^{25}$ Im slowakischen Cantus Catholici kommt ähnlich dem tropierten Kyrie Magne Deus auch das Sanctus bei den allgemeinen Festgesängen vor, hier wurde die Sanctus-Melodie aber nur einmal und mit einem Text gedruckt. ${ }^{26}$ Nach dem Oktav-Mittelschluss des ungarischen und slowakischen Sanctus, der einem identischen Melodieverlauf folgt, biegt im slowakischen Sanctus anders als im ungarischen die Melodie wieder nach oben, und damit entsteht gegenüber der Melodie der ungarischen Graduale (und natürlich der mittelalterlichen Quel$15-28$

22. Benjamin Rajeczky, „Spätmittelalterliche Organalkunst in Ungarn“, Studia Musicologica 1 (1961),

23. Für Beispiele u. a. aus den humanistischen metrischen Oden, dem Eperieser Gradual, dem Tabulaturbuch Vietoris und dem Kájoni Kodex siehe Pál Richter, „Quint- und Oktavparallelen in den Handschriften mit Orgelbegleitung des 17. Jahrhunderts“, Ars Organi 49 (2001), 19-26.

24. Vgl. Ordinariums-Gesänge in Mitteleuropa, Sanctus 39-194, Agnus 56. Die ungarischsprachigen Vorkommen s. Batthyány-Gradual, p. 383; Spáczay-Gradual, p. 218; Patay-Gradual, f. 132; Kálmáncsai Gradual, f. 140; Béllyei Gradual, p. 142; Öreg Gradual, p. 152; die Beschreibung der Quellen siehe A magyar kéziratos énekeskönyvek és versgyüjtemények bibliográfiája (1542-1840) [Bibliographie der ungarischen handschriftlichen Gesangbücher und Gedichtsammlungen (1542-1840)], hrsg. von Béla Stoll (Budapest: Balassi Kiadó, 22002), Nr. 6, 34, 12, 41, 68 sowie Régi Magyarországi Nyomtatványok 3. 1636-1655, Nr. 1643.

25. Die Variante des Batthyány-Graduals gemäß dem Spáczay- und dem Öreg Gradual korrigiert siehe Graduale Ráday saeculi XVII, hrsg. von Ilona Ferenczi (Budapest: MTA Zenetudományi Intézet, 1998) (= Musicalia Danubiana 16), Anhang Nr. 276, 330-331. Die Variante im Kálmáncsai Gradual herausgegeben in Kálmáncsai graduál. 1622-1626, hrsg. von Ilona Ferenczi und Gábor Pap (Kecskemét: Nemzeti Kincseinkért Egyesület, 2005), Nr. 287.

26. Erschienen in Burlas-Fišer-Hořejš, Hudba na Slovensku, Cantus Catholici Nr. 159. 
Notenbeispiel 4 Oben: Sanctus im Cantus Catholici (1655)

Unten: Sanctus im Batthány-Gradual (Anfang des 17. Jahrhunderts)
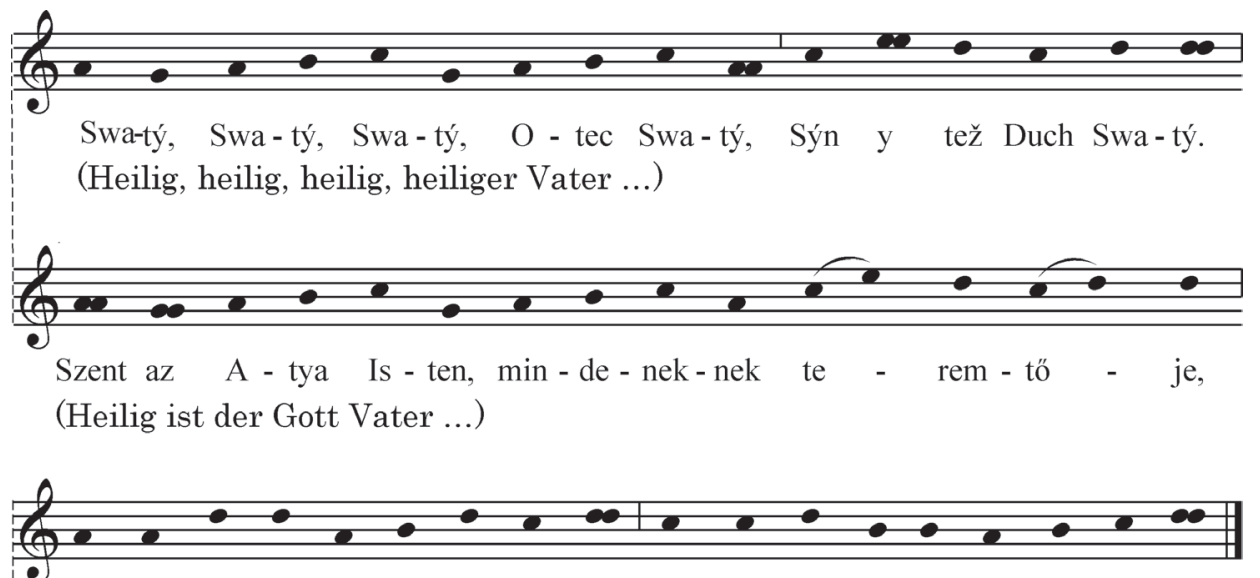

Wsse ge - di - ný, Hos-po-d’in mi - lý. Stwor-ce, zbaw-ce a wy-kú - pe-ňý.

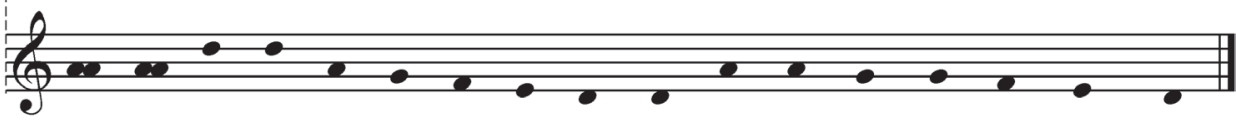

Szent a Fi - ú Is - ten a mi meg - vál - tó U - runk, Jé - zus Krisz - tus.

len) eine organale Variante. Während sich der tropierte Text des slowakischen Cantus Catholici gut der originalen Melodiestruktur anpasst, lässt der ungarische Text - obwohl er sich der Hauptmelodie beigesellt - die Zeilengränzen verblassen (Notenbeispiel 4).

In den ungarischen Quellen findet sich auch in diesem Fall keine Spur einer echten mehrstimmigen Bearbeitung, wohl aber gibt es in aus Böhmen stammenden und Ordinariumssätze enthaltenden Handschriften sowie in utraquistischen Gradualen reichlich Angaben für Melodienverzierung in drei- und vierstimmiger Organumtechnik. Während sich ein Teil der lateinischen Sanctus-Organa auf die Melismen dreimaliger Anrede erstreckt, repräsentiert in den utraquistischen Gradualen des 16. Jahrhunderts das tropierte Sanctus in tschechischer Sprache den syllabischen Stil. ${ }^{27}$

27. Der Text der beiden Sanctus-Tropen: Wssemohúcý Bůh stwořitel und Každý duch chwal Boha wssemohúcý. Die Incipits der Organa siehe Handschriften mit mehrstimmiger Musik, 143, 165, 241, 250, 252, 253, 276, 284 und 301 (alle böhmische Quellen), ein zweistimmiges Organum aus Zwickau siehe auf Seite 410. - In Kájonis ORGANO MISSALE, 382-386 (Aliud Sanctus und Agnus ad Rorate de B. Virg.) kommt nur die vox principalis mit Begleitung vor. 
Nicht nur in den rhythmisch ungebundenen gregorianischen Sätzen, sondern auch in den einstimmigen ungarischen Gemeindeliedern des 16. Jahrhunderts sind Reminiszenzen einer einstigen organalen Technik zu entdecken. Gál Huszár widmet in seinem ersten Gesangbuch (1560-1561) - von dem wir wissen, dass es viele Melodien solcher Lieder bewahrte, welche die übrigen Gesangbuch-Redakteure des 16. Jahrhunderts oder er selbst 1574 nur mit den Texten herausgaben - ein gesondertes Kapitel den nach Psalmen gedichteten Liedern. Psalm 6 der Lobgesänge aus den Psalmen, die Psalmenparaphrase von Gergely Szegedi (Szent Dávid király bünei ellen - Gegen seine Sünden der König David), erweckt Aufmerksamkeit mit seiner sonderbaren Zeilenstruktur und Melodie: jede der $3 \times 3$ Zeilen der Strophen gliedert sich in Teile von 5+5+6 Silben. ${ }^{28}$ Abgesehen davon, dass der Rhythmus in vier von den neun kurzen Zeilen korrumpiert und die Töne 6-9 der ersten Zeile vermutlich eine Sekunde tiefer gedruckt wurden, ${ }^{29}$ ist in der dritten großen Zeile gegen Ende des Liedes zu spüren, dass die Melodieführung nicht homogen ist, als wähle sie von hier und dort je ein Segment: Das erste Glied der dritten großen Zeile bringt die Melodie der ersten Zeile eine Quinte tiefer und setzt sie dann ungewohnterweise nach einem Sextsprung fort. ${ }^{30}$ Das entstandene Mangelempfinden bezüglich dieser Melodie verstärkt sich noch, wenn man die fast zweihundert Jahre später herausgegebene vollkommenere Variante kennenlernt. - Nach ähnlich strukturiertem Muster entstand Mátyás Nagybánkais Paraphrase von Psalm 71 Ne hagyj elesnem, felséges Isten (Lass mich nicht stürzen, hochheiliger Gott) 1575, die aber mit Melodie erst 1744 in Klausenburg (Kolozsvár, heute Cluj-Napoca) erschien. ${ }^{31}$ Diese Melodie ist viel ausgeglichener als ihr Vorgänger aus dem 16. Jahrhundert, und auch die dritte große Zeile beginnt nicht mit dem Herumtappen um den Grundton. Untersucht man beide Melodien gemeinsam, erhält man gegen Ende der ersten groBen und in der zweiten Zeile Zweistimmigkeit im Organumstil (Notenbeispiel 5). ${ }^{32}$

Das anschaulichste Beispiel bietet der Vergleich je einer Melodie aus dem ungarischsprachigen Cantus Catholici (1651) und dem Klausenburger Gesangbuch (1744). Die Melodien waren bereits im 16. Jahrhundert bekannt. ${ }^{33}$ Die erste, Könyörögjünk az Istennek, Szentlelkének (Nun lasset uns Gott, Gott den heilgen Geist anflehn), ist ein Lied von Gál Huszár, von dem er in seinen beiden Gesangbüchern (1560-1561, 1574) ausschließlich den Text gedruckt hat. Die Melodie wurde - obwohl sie bei mehreren Liedern als Muster, als Notenanzeige angegeben

28. Siehe Huszár - Kálmáncsehi, f. P7v; Textquellen siehe RPHA Nr. 1306.

29. Im 3. Notenbeispiel ist die Melodie originalgetreu wiedergegeben, dagegen wurde der Rhythmus der Zeilen vereinheitlicht.

30. Da die dritte Zeile um eine Quinte tiefer als die erste beginnt, ist es anzunehmen, dass dies - wie früher bei der mittelalterlichen Sequenz - aus einem Quintorganum stammt.

31. Siehe Kálmán Csomasz Tóth, A XVI. század magyar dallamai [Ungarische Melodien des 16. Jahrhunderts], (im Weiteren RMDT I) (Budapest: Akadémiai Kiadó, 1958) (=Régi Magyar Dallamok Tára 1), Nr. 196/ III; Textquellen vgl. RPHA Nr. 1052.

32. Die Melodien sind mit auf Viertel reduzierten Werten wiedergegeben.

33. Siehe RMDT I, Nr. 105/I, II. 
war $^{34}$ - erst in der Mitte des 17. Jahrhunderts gedruckt. Während Gál Huszár die Bitte an Gott den Heiligen Geist vor dem Abendmahl als liturgische Stelle des Liedes angibt, setzte man es nach dem Cantus Catholici vor die Predigt. ${ }^{35}$ Der

NotenBeISPIEL 5 Oben: Fürbitte im Klausenburger Gesangbuch (1744)

Unten: Psalm im Gesangbuch von Gál Huszár (1560-1561)


Te szent Fi - a - dért légy se-gít-ség-gel, ne es-sem két-ség-ben;
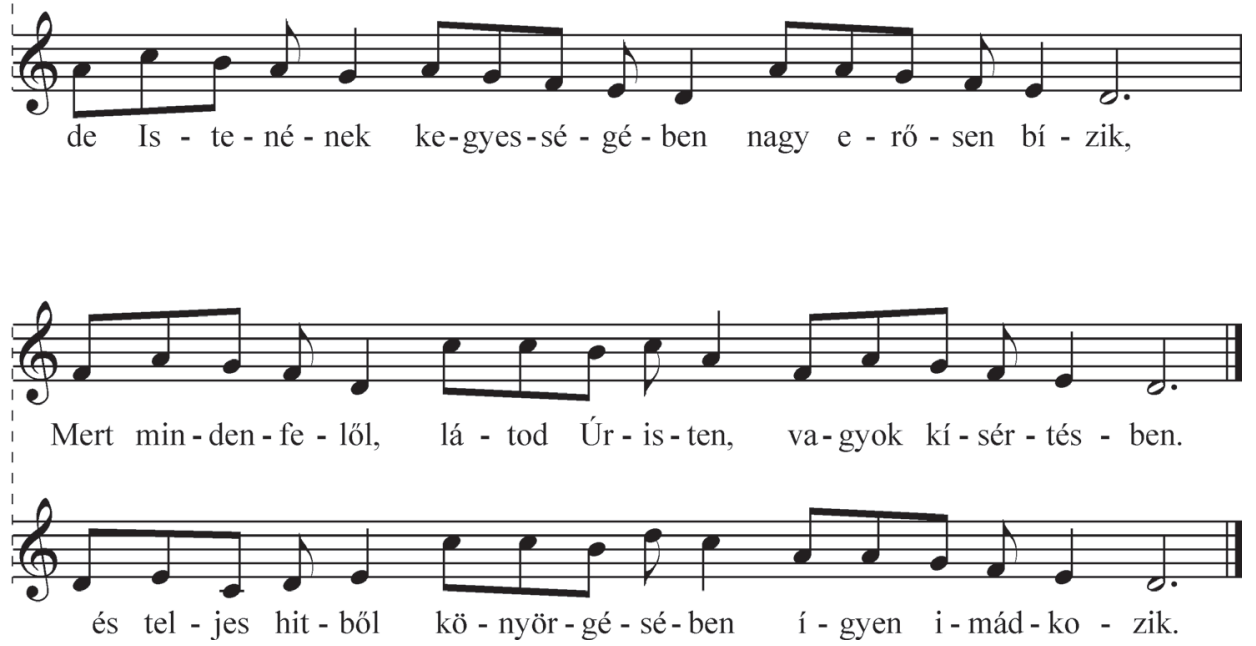

34. Vgl. RMDT I, 651; Textquellen s. RPHA Nr. 849.

35. Huszár, A keresztyéni gyülekezetben való isteni dicséretek, Kálmáncsehi, Reggeli éneklések, 15601561 ..., f. M4v: Precatio ad Deum Spiritum sanctum ante sacram Contionem; Huszár, A keresztyéni gyülekezetben való isteni dicséretek és imádságok, Komjáti 1574 f. 319: „von Gál Huszár“. Die Beschreibung des ungarischen Gesangbuches Cantus Catholici (1651) siehe Régi Magyarországi Nyomtatványok 3, Nr. 2381; die Textquellen s. RPHA Nr. 774. 
Notenbeispiel 6a Fürbitte im Cantus Catholici (1655)

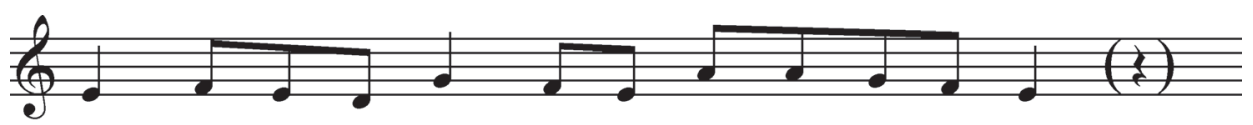

Kö - nyö - rög - jünk az Is - ten - nek, szent lel - ké - nek,

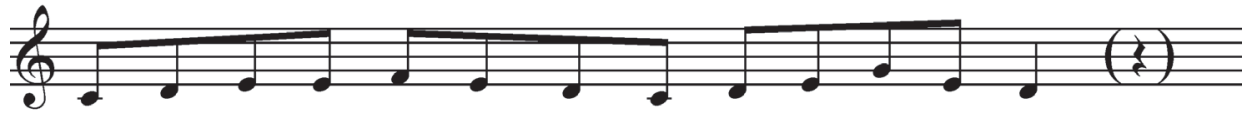

Bo - csás - sa ki ma - gas menny - ből az ő vi - lá - gát,



Ve - gye el mi szí - vünk-nek min - den ho - má - lyát,

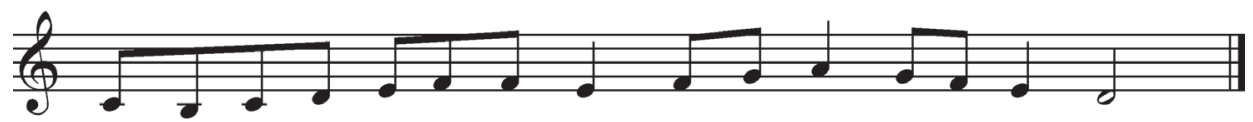

Hogy ért - hes - sük Is - te - nünk-nek min-den-ben a - ka - rat - ját.

NotenBEISPIEL 6b Loblied im Klausenburger Gesangbuch (1744)



Ma - gasz - tal - juk, ke - resz - tyé - nek, az Úr - is - tent,

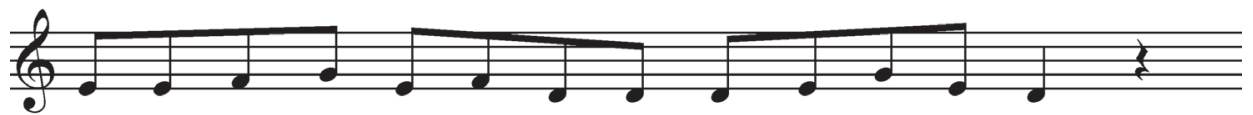

Ki né - künk meg - ke - gyel - me - zett nagy jó - vol - tá - ért,

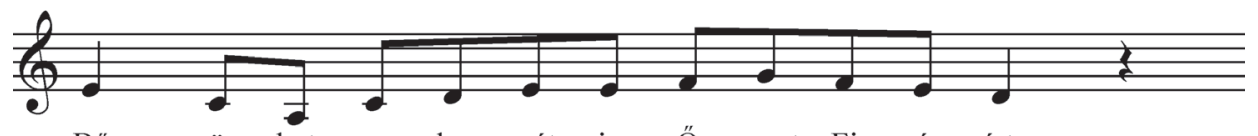

Bű - nün - ket meg - bo - csát - ja Ö szent Fi - á - ért,



És ö - rök é - le - tet í - gér csak a Jé - zus Krisz-tu - sért. 
Textdichter des anderen Liedes, Magasztaljuk, keresztyének, az Úristent (Christenleute, lasset uns den Herrgott preisen), ist unbekannt. ${ }^{36}$ Die vierzeilige Struktur der beiden Lieder scheint beim ersten Anschauen ausgeglichen zu sein, doch unterscheidet sich die innere Gliederung der Zeilen mit geringer Silbenzahlabweichung erheblich voneinander (Notenbeispiel 6). ${ }^{37}$

Es fällt auf, dass in den Zeilen 1, 2 und 4 beide Lieder ähnlich gestaltet sind, ${ }^{38}$ die jeweiligen dritten Zeilen ergänzen sich jedoch wie Eröffnungs- und Schlussformeln der mittelalterlichen zweistimmigen Organa mit Gegenbewegung (Notenbeispiel 7).

Notenbeispiel 7 Die Melodie von Cantus Catholici (1655)

zusammen mit der Melodie vom Klausenburger Gesangbuch (1744)



Die uns unbekannte Urform des Liedes aus dem 16. Jahrhundert entstammte wahrscheinlich einer konkreten zweistimmigen Klangerfahrung, aus der die zwei Varianten aus dem 17. und 18. Jahrhundert entstehen konnten. Welche von beiden kann die Hauptmelodie und welche die Begleitstimme gewesen sein? Oder kamen vielleicht alle beide als Hauptmelodie vor? Da man in mehreren Fällen die Erfahrung macht, dass die Klausenburger und Debreziner Gesangbücher des 18. Jahrhunderts genauer den Melodien des 16. Jahrhunderts folgen als die katholischen Gesangbücher des 17. Jahrhunderts, halten wir es für wahrscheinlich, dass die zweite, die nach unten biegende Melodieform die frühere gewesen ist. Auch gleicht den Tonbestand der ersten Melodiehälfte die nach unten biegende zweite Hälfte besser aus.

Unter den ungarischen Melodien des 16.-18. Jahrhunderts gibt es demnach einige, die durch ihre Varianten auf eine in Ungarn jahrhundertelang erhalten gebliebenen mehrstimmigen Gesangspraxis hinzuweisen scheinen. ${ }^{39}$ Bei einem so ausdauernden Gebrauch der organalen Mehrstimmigkeit ist die Annahme wohl nicht unbegründet, dass diese Technik in Ungarn weit verbreitet war.

36. Die zehn Töne des Liedes mit weiteren Varianten finden sich in den 1774 und 1778 herausgegebenen Debrecener Gesangbüchern.

37. $12(4,4,4), 13(8,5), 12(7,5), 15(8,7)$. - Die Melodien sind mit auf Viertel reduzierten Werten wiedergegeben. Die Melodien der drei Jahrzehnte nach dem Klausenburger Gesangbuch erschienenen Debrecener Gesangbücher konnten hier nicht berücksichtigt werden.

38. Allerdings ist zuweilen auch in diesen Zeilen der mehrstimmige Hintergrund erkennbar, z. B. gegen Ende der 1. Zeile in den Tönen 8-11. - Weitere Beispiele für einen vermutlichen organalen Hintergrund: Mindenek meghallják, RMDT I, Nr. 136/I, II und RMDT I, Nr. 43 bzw. 44; Jer, mindnyájan örüljünk, RMDT I, Nr. 129/I.

39. Der retrospektive organale Stil ist nicht nur im ungarischen, sondern - wie gesehen - auch im deutschen und böhmischen Gebrauch bis zum Ende des Mittelalters in Klöstern, Abteien und einzelnen Pfarrkirchen sowie Fraternitäten erhalten geblieben; vgl. das Vorwort von W. Dömling im Übertragungsband von Die Handschrift London, VII. 